\section{CHRONIC OSTEOMYELITIS IN A PATIENT FROM GAMBIA}

M Benavides-Nieto', M Agud-De Dios', A Vázquez-Pérez', J Bustamante', J Cabello-Blanco², F Baquero-Artigao', MJ Mellado-Peña', T Sáinz-Costa', M García López-Hortelano'

'Hospital La Paz, General Paediatrics and Infectious and Tropical Diseases Department, Madrid, Spain. ${ }^{2}$ Hospital La Paz, Orthopaedic Surgery and Traumatology Department, Madrid, Spain.

\section{BACKGROUND}

- Chronic osteomyelitis is a pathology characterized by a long-standing and recurrent clinical course; especially significant in developing countries.

- Its management usually requires both antibiotic and surgical treatment.

\section{CASE PRESENTATION}

\section{7-year-old patient from Gambia}

- 7-year history of bone pain in left lower limb (LLL) associated with abscessed lesions.

- Two surgical interventions and a 6-month course of parenteral antibiotics was administered in his country of origin.

- Exacerbations with discharge of purulent material along multiple fistulous paths treated with oral antibiotics.

- No BCG vaccination, no other symptoms, no significant previous history.
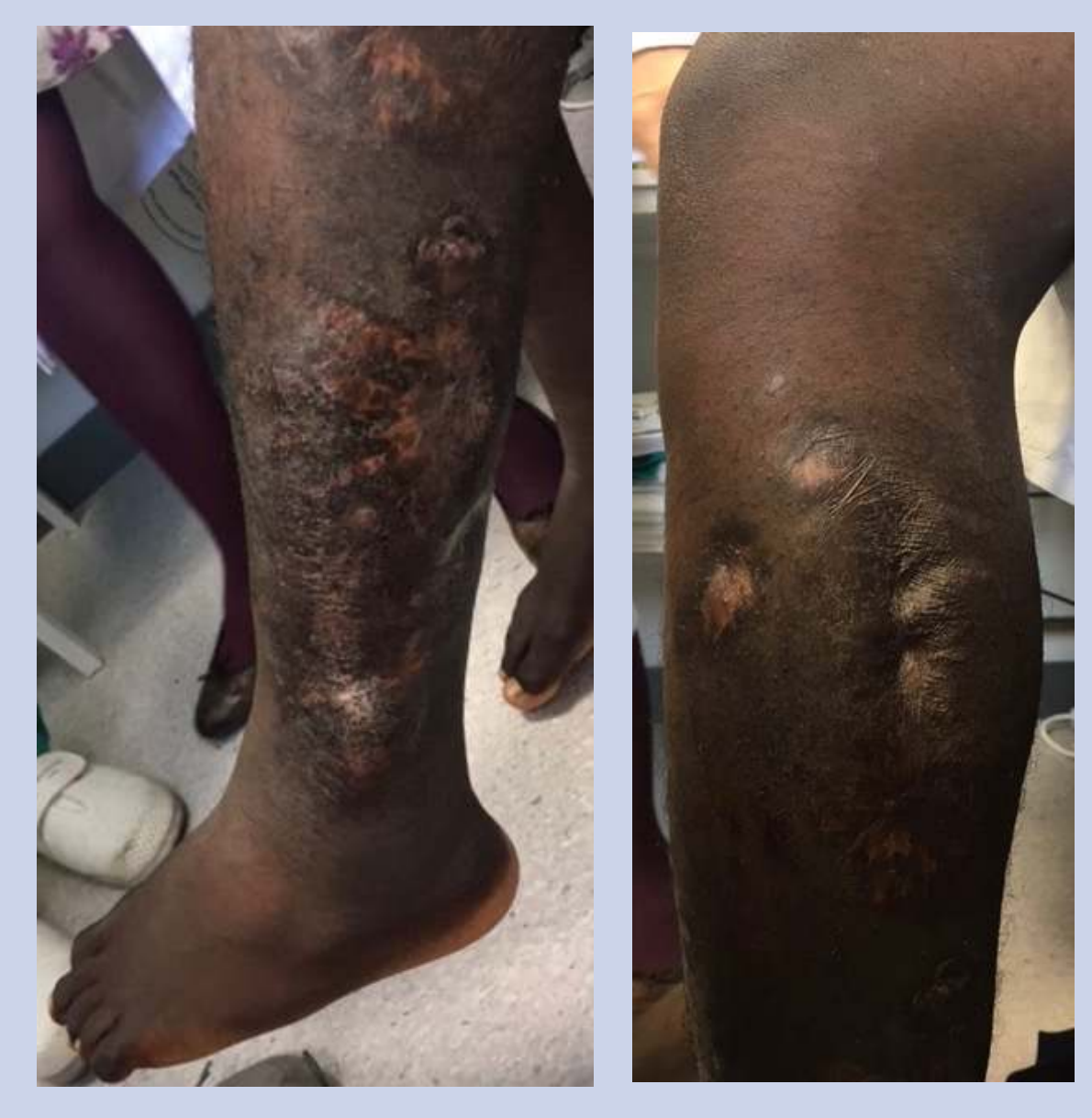

- CRP 12,5mg/l, ESR 58mm, fibrinogen $569 \mathrm{mg} / \mathrm{dl}$.

- Normal CBC, coagulation and other biochemistry tests

- Mantoux 0mm, negative Quantiferon

- Negative thick blood smear and PCR for Plasmodium

- Negative blood, urine, stool cultures, and parasites tests.

- Negative HIV, Syphilis, Schistosoma, Strongyloides, Toxocara canis serologies

- Chest Xray.: normal

- LLL Xray: increased fibular radiolucency without corticomedullary differentiation, and irregular contours.

- MRI findings: suggestive of chronic osteomyelitis with diffuse affectation of the fibula and formation of bone sequestration and sewers, extension of inflammation to soft tissues and collections in muscular compartment.

- Bone scintigraphy didn't show other foci.

Given the suspicion of chronic osteomyelitis, surgery was permormed with:

$>$ resection of peroneal diaphysis

$>$ fistulectomy

$>$ debridement of left lower limb

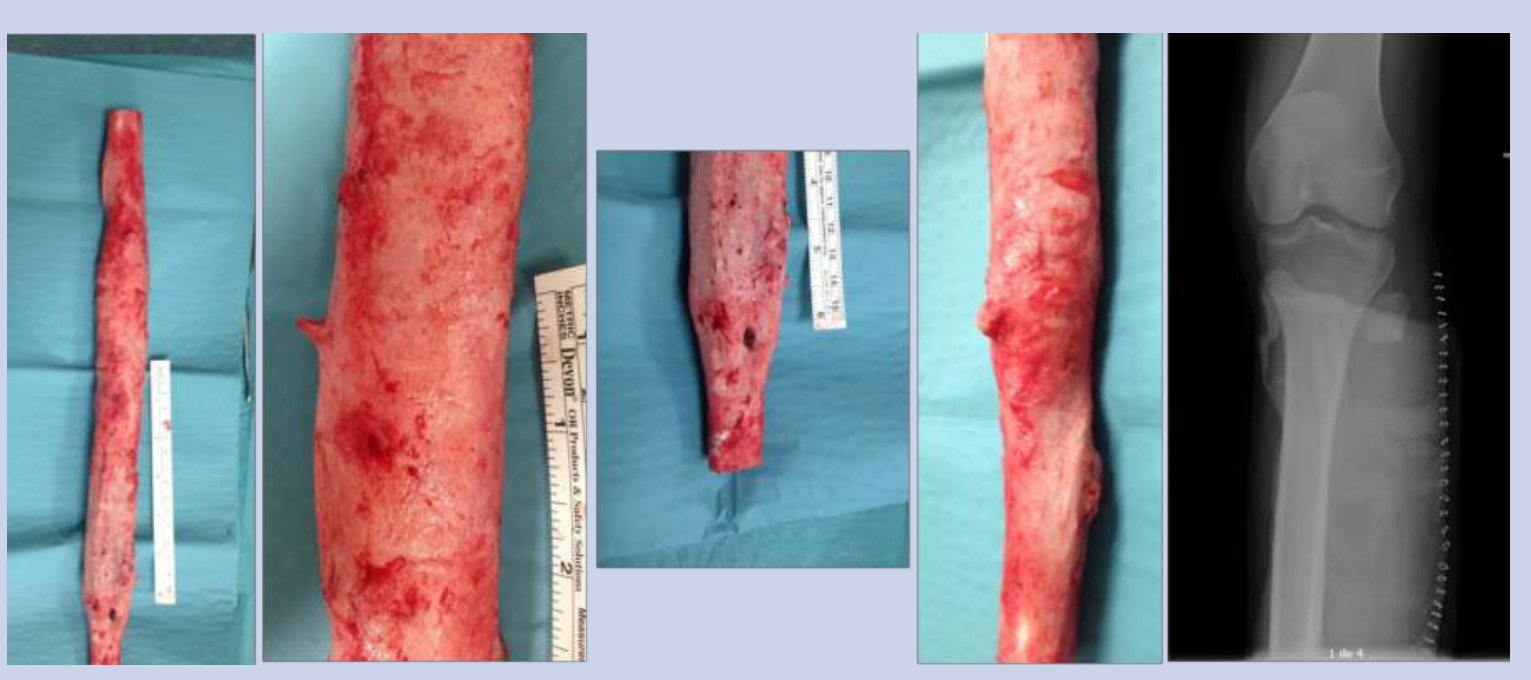

- Anatomopathological findings compatible with chronic osteomyelitis.

- Isolation in the biopsy: Methicillin-sensitive Staphylococcus aureus

- PCR Mycobacterium and other cultures: negative

\section{Antibiotic treatment:}

Parenteral rifampicin and cefazolin (14days) $\quad+$ oral cloxacillin and rifampicin (9months)

\section{DISCUSSION}

- Chronic osteomyelitis is a complex pathology, which requires a multidisciplinary approach; both surgical treatment and microbiological diagnosis are fundamental to optimize the treatment. 